\title{
Inductance extraction of Press-Pack IGBT by Considering Displacement Current
}

\author{
Jinyuan $\mathrm{Li}^{1}$,Zhongyuan $\mathrm{Chen}^{1}$,Lei Zhang ${ }^{1}$, Chouwei $\mathrm{NI}^{2}$ and Zhibin $\mathrm{ZHAO}^{2}$ \\ ${ }^{1}$ State Key Laboratory of Advanced Power Transmission Technology (Global Energy Interconnection Research Institute of State Grid \\ Corporation of China), Beijing 102209, China \\ ${ }^{2}$ State Key Laboratory of Alternate Electrical Power System with Renewable Energy Sources (North China Electric Power University), \\ Beijing 102206, China)
}

\begin{abstract}
Taking the displacement current generated by charges accumulation into consideration, this paper presents a inductance extraction method for press-pack IGBT where conductor might not form a closed loop in simulation. Charge accumulating on ends of conductor is considered and then Ampere's circuital law (ACL) could be meet. Vector potential A also could be affected by displacement current, which leads to a different formulation of partial inductance. A simplified model based on practical structure are used in numerical experiment for comparison between existing method and proposed method.
\end{abstract}

\section{Introduction}

The press-pack IGBT has become much more attractive than ever due to its high voltage and high current ratings. In the switching process, the maximum current overshoot of paralleled chips inside PPI decides the application limit of the device, which is directly affected by stray parameters of the package module.

The inductance in package is important for current distribution between chips, which mainly exists in the conductors of package. The magnetic coupling effect between conductor segments that don't form a closed loop needs to be considered because the return current path might be distant.

Therefore, Ruehli [1] proposed the definition of partial inductance based on flux linkage method and gave a series of formulas to establish the relationship between incomplete loops and closed loops. In recent years, Paul [2] and Holloway [3] made some detailed analysis and summary. Afterwards, the partial element equivalent circuit (PEEC) method that based on the partial inductance theory is widely applied [4-6].

To obtain partial inductance, magnetic vector potential $\boldsymbol{A}$ generated by each conductor segment needs to be calculated at first. Kalhor's study [7] showed that two charges accumulating with time need to be placed at both ends of a conductor, which generate displacement current in space, to meet Ampere's circuital law (ACL) in incomplete loop. Kalhor [8] pointed out that Biot-Savart's Law is still applicable in this situation, but effect on $\boldsymbol{A}$ is not analyzed. According to (1), differential form of ACL is applied in the calculation of $\boldsymbol{A}$, which means total current that includes displacement current is used.

$$
\boldsymbol{A}(\boldsymbol{r})=\frac{1}{4 \pi} \int_{V^{\prime}} \frac{\nabla^{\prime} \times \boldsymbol{B}\left(\boldsymbol{r}^{\prime}\right)}{\left|\boldsymbol{r}^{\prime}-\boldsymbol{r}\right|} \mathrm{d} V^{\prime}
$$

Hence, this paper presents an inductance extraction method of partial inductance calculation by considering both conduction current and displacement current. In section II, $\boldsymbol{A}$ is derived when charges accumulating at both ends of a conductor. Section III gives a method of partial inductance calculation based on the result of $\boldsymbol{A}$. Section IV makes a numerical experiment for a simplified structure and show difference of results between methods before and after considering displacement current.

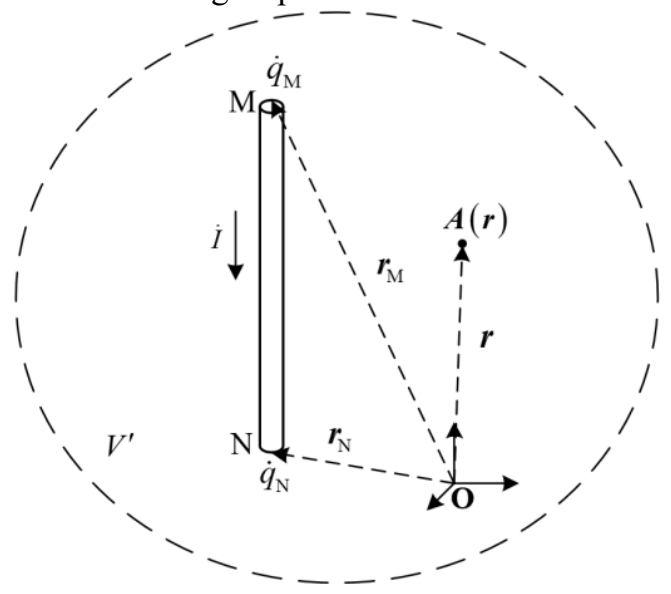

Fig. 1. Conductor segment carrying current in space.

2 A Generated by Displacement Current 
As shown in Fig. 1, when a conductor segment carries alternating current $\dot{I}$, opposite charges accumulate respectively at both ends of the conductor, noted as points $\mathrm{M}$ and $\mathrm{N}$, and generate displacement current in space. The opposite charges are noted as $\dot{q}_{\mathrm{M}}$ and $\dot{q}_{\mathrm{N}}$. According to current continuity equation, (2) is given.

$$
-j \omega \dot{q}_{\mathrm{A}}=j \omega \dot{q}_{\mathrm{B}}=\dot{I}
$$

As mentioned above, ACL is satisfied in this model. So (4) is obtained by substitute (3) into (1).

$$
\begin{gathered}
\nabla \times \dot{\boldsymbol{B}}=\dot{\boldsymbol{J}}_{\mathrm{C}}+\dot{\boldsymbol{J}}_{\mathrm{D}} \\
\dot{\boldsymbol{A}}(\boldsymbol{r})=\frac{\mu_{0}}{4 \pi} \int_{V^{\prime}} \frac{\dot{\boldsymbol{J}}_{\mathrm{C}}\left(\boldsymbol{r}^{\prime}\right)+\dot{\boldsymbol{J}}_{\mathrm{D}}\left(\boldsymbol{r}^{\prime}\right)}{\left|\boldsymbol{r}^{\prime}-\boldsymbol{r}\right|} \mathrm{d} V^{\prime}
\end{gathered}
$$

where $V^{\prime}$ represents the whole area of source, $\boldsymbol{r}^{\prime}$ and $\boldsymbol{r}$ are space vectors pointing to source point and field point, respectively. $\dot{\boldsymbol{J}}_{\mathrm{C}}$ is current density of conduction current $\dot{I}_{\mathrm{C}} \cdot \dot{\boldsymbol{J}}_{\mathrm{D}}$ is sum current density of displacement current generated by $\dot{q}_{\mathrm{M}}$ and $\dot{q}_{\mathrm{N}}$, and can be calculated by (5). $\dot{\boldsymbol{D}}$ represents electric displacement vector.

$$
\begin{gathered}
\dot{\boldsymbol{J}}_{\mathrm{D}}\left(\boldsymbol{r}^{\prime}\right)=j \omega \dot{\boldsymbol{D}}\left(\boldsymbol{r}^{\prime}\right) \\
\dot{\boldsymbol{D}}=\dot{q} \boldsymbol{r}_{\mathrm{q}} /\left(4 \pi r_{\mathrm{q}}^{3}\right)
\end{gathered}
$$

According to Gauss' law, $\dot{\boldsymbol{D}}$ generated by a charge in space is given in (6), where $\boldsymbol{r}_{\mathrm{q}}$ is space vector pointing from charge to field point. $\dot{\boldsymbol{J}}_{\mathrm{D}}$ consists of two parts, $\dot{\boldsymbol{J}}_{\mathrm{DM}}$ and $\dot{\boldsymbol{J}}_{\mathrm{DN}}$, due to different charges $\dot{q}_{\mathrm{M}}$ and $\dot{q}_{\mathrm{N}}$. Combine (5) and (6),

$$
\begin{aligned}
\dot{\boldsymbol{J}}_{\mathrm{D}}\left(\boldsymbol{r}^{\prime}\right) & =\dot{\boldsymbol{J}}_{\mathrm{DA}}\left(\boldsymbol{r}^{\prime}\right)+\dot{\boldsymbol{J}}_{\mathrm{DB}}\left(\boldsymbol{r}^{\prime}\right) \\
& =\frac{-\dot{I}}{4 \pi}\left(\frac{\boldsymbol{r}^{\prime}-\boldsymbol{r}_{\mathrm{M}}}{\left|\boldsymbol{r}^{\prime}-\boldsymbol{r}_{\mathrm{M}}\right|^{3}}-\frac{\boldsymbol{r}^{\prime}-\boldsymbol{r}_{\mathrm{N}}}{\left|\boldsymbol{r}^{\prime}-\boldsymbol{r}_{\mathrm{N}}\right|^{3}}\right)
\end{aligned}
$$

where $\boldsymbol{r}_{\mathrm{M}}$ and $\boldsymbol{r}_{\mathrm{N}}$ are space vectors pointing to $\mathrm{M}$ and $\mathrm{N}$, respectively. Thus, sum $\dot{\boldsymbol{A}}$ in (4) can be also spited into two items $\dot{\boldsymbol{A}}_{\mathrm{C}}$ and $\dot{\boldsymbol{A}}_{\mathrm{D}}$, corresponding to current density. Therefore, $\dot{\boldsymbol{A}}_{\mathrm{D}}$ can be calculated as followed.

$$
\begin{aligned}
& \dot{\boldsymbol{A}}_{\mathrm{D}}(\boldsymbol{r})=\frac{\mu_{0}}{4 \pi} \int_{V^{\prime}} \frac{\dot{\boldsymbol{J}}_{\mathrm{D}}\left(\boldsymbol{r}^{\prime}\right) d V^{\prime}}{\left|\boldsymbol{r}^{\prime}-\boldsymbol{r}\right|} \\
& =\frac{\mu_{0} \dot{I}}{16 \pi^{2}} \int_{V^{\prime}} \frac{-1}{\left|\boldsymbol{r}^{\prime}-\boldsymbol{r}\right|}\left[\frac{\left(\boldsymbol{r}^{\prime}-\boldsymbol{r}_{\mathrm{M}}\right)}{\left|\boldsymbol{r}^{\prime}-\boldsymbol{r}_{\mathrm{M}}\right|^{3}}-\frac{\left(\boldsymbol{r}^{\prime}-\boldsymbol{r}_{\mathrm{N}}\right)}{\left|\boldsymbol{r}^{\prime}-\boldsymbol{r}_{\mathrm{N}}\right|^{3}}\right] d V^{\prime} \\
& =\dot{\boldsymbol{A}}_{\mathrm{DM}}(\boldsymbol{r})+\dot{\boldsymbol{A}}_{\mathrm{DN}}(\boldsymbol{r})
\end{aligned}
$$

Similarly, $\dot{A}_{\mathrm{D}}$ has two parts $\dot{\boldsymbol{A}}_{\mathrm{DM}}$ and $\dot{\boldsymbol{A}}_{\mathrm{DN}}$ generated by $\dot{q}_{\mathrm{M}}$ and $\dot{q}_{\mathrm{N}}$. Hence formulation after derivation is shown.

$$
\dot{\boldsymbol{A}}_{\mathrm{DM}}\left(\boldsymbol{r}_{1}\right)=-\mu_{0} \dot{\boldsymbol{I}}_{r_{1}} /(8 \pi)
$$

Equation (12) indicates magnitude of $\dot{\boldsymbol{A}}_{\mathrm{DM}}$ is constant in space, and direction is in accordance with $\boldsymbol{r}_{1}$. We can easily derive that $\dot{A}_{\mathrm{DM}}$ is irrotational.

$$
\nabla \times \dot{A}_{\mathrm{DM}}=0
$$

In the same way, $\dot{\boldsymbol{A}}_{\mathrm{DN}}$ generated by another charge (3) $\dot{q}_{\mathrm{N}}$ can be calculated by (14), where $\boldsymbol{r}_{2}$ is space vector pointing to field point from point N.

$$
\dot{\boldsymbol{A}}_{\mathrm{DN}}\left(\boldsymbol{r}_{2}\right)=\mu_{0} \dot{\boldsymbol{I}}_{r_{2}} /(8 \pi)
$$

Consequently, sum $\dot{\boldsymbol{A}}$ consists of three parts, $\dot{\boldsymbol{A}}_{\mathrm{C}}$, $\dot{A}_{\mathrm{DM}}$, and $\dot{\boldsymbol{A}}_{\mathrm{DN}}$, and has a different direction with conduction current.

$$
\begin{aligned}
& \dot{\boldsymbol{A}}=\dot{\boldsymbol{A}}_{\mathrm{C}}+\dot{\boldsymbol{A}}_{\mathrm{D}}=\dot{\boldsymbol{A}}_{\mathrm{C}}+\dot{\boldsymbol{A}}_{\mathrm{DM}}+\dot{\boldsymbol{A}}_{\mathrm{DN}} \\
& =\dot{\boldsymbol{A}}_{\mathrm{C}}+\mu_{0} \dot{I}\left(-\boldsymbol{e}_{\boldsymbol{r}-\boldsymbol{r}_{\mathrm{M}}}+\boldsymbol{e}_{\boldsymbol{r}-\boldsymbol{r}_{\mathrm{N}}}\right) /(8 \pi)
\end{aligned}
$$

When any two conductor segments with same current $\dot{I}$ are connected at the end, as shown in Fig. 2,

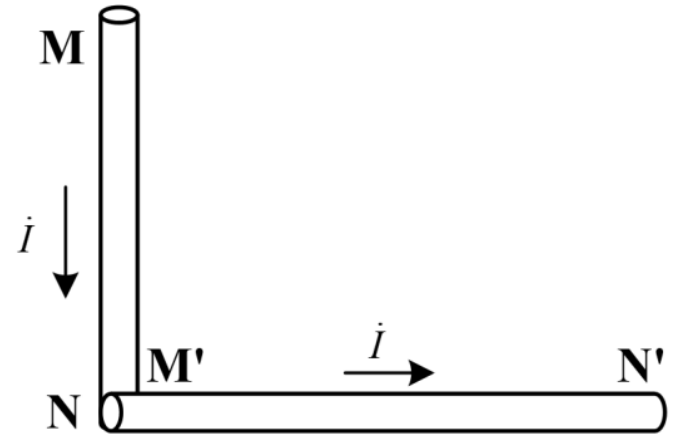

Fig. 2. Connection of two conductors.

The four end points are noted as $\mathrm{M}, \mathrm{N}, \mathrm{M}^{\prime}$, and N', where $\mathrm{N}$ and $\mathrm{M}^{\prime}$ is connected. On the joint, because opposite charges accumulate in the same location and generate opposite displacement current, they generate opposite $\boldsymbol{A}$ in space as (16).

$$
\dot{\boldsymbol{A}}_{\mathrm{DN}}=-\dot{\boldsymbol{A}}_{\mathrm{DM}^{\prime}}
$$

where $\dot{\boldsymbol{A}}_{\mathrm{DN}}$ and $\dot{\boldsymbol{A}}_{\mathrm{DM}^{\prime}}$ are generated respectively by charges accumulating on point $\mathrm{N}$ and $\mathrm{M}^{\prime}$. Therefore effect of them is compensated. $\operatorname{Sum} \boldsymbol{A}$ is

$$
\begin{aligned}
& \dot{\boldsymbol{A}}=\dot{\boldsymbol{A}}_{\mathrm{C}}+\dot{\boldsymbol{A}}_{\mathrm{D}} \\
& =\dot{\boldsymbol{A}}_{\mathrm{C}}+\dot{\boldsymbol{A}}_{\mathrm{DM}}+\dot{\boldsymbol{A}}_{\mathrm{DN}}+\dot{\boldsymbol{A}}_{\mathrm{DM}^{\prime}}+\dot{\boldsymbol{A}}_{\mathrm{DN}} \\
& =\dot{\boldsymbol{A}}_{\mathrm{C}}+\dot{\boldsymbol{A}}_{\mathrm{DM}}+\dot{\boldsymbol{A}}_{\mathrm{DN}^{\prime}}
\end{aligned}
$$

Similarly, when conductor segments form a closed loop, all effect of displacement can be compensated based on current continuity equation. That means the introduced item $\dot{\boldsymbol{A}}_{\mathrm{D}}$ will not influence any results of closed loops by existing method, so it can be also applied in a closed loop. But $\dot{A}_{\mathrm{D}}$ may modify result of partial inductance for conductor segments. 


\section{Partial Inductance Calculation}

Existing method of partial inductance in [4] assumed that conductor segment $\mathrm{S} 1$ as a portion of half-infinite loop in Fig.

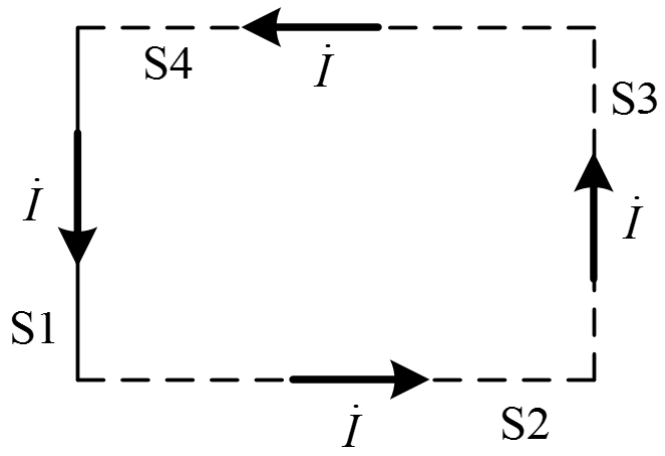

Fig. 3. Conductor segment S1 in a half-infinite loop.

where segments 2 and 4 have infinite length. Partial inductance can be calculated by (18) based on existing method. $\boldsymbol{A}_{\mathrm{C} i}$ is generated by conduction current in segment $\mathrm{S} i$.

$$
\begin{aligned}
L_{\mathrm{p} 11} & =\frac{1}{\dot{I}} \oint \dot{\boldsymbol{A}}_{\mathrm{C} 1} \cdot \mathrm{d} \boldsymbol{l}=\frac{1}{\dot{I}} \sum_{i=1}^{4} \int_{\mathrm{S} i} \dot{\boldsymbol{A}}_{\mathrm{C} 1} \cdot \mathrm{d} \boldsymbol{l} \\
& =\frac{1}{I} \int_{\mathrm{S} 1} \boldsymbol{A}_{\mathrm{C} 1} \cdot \mathrm{d} \boldsymbol{l}+0+0+0
\end{aligned}
$$

Considering displacement current, because direction of $\boldsymbol{A}_{\mathrm{D}}$ is different with current, last three parts of integral in (18) is not all of zero. So partial inductance of conductor segments is redefined as followed.

$$
\begin{aligned}
& \frac{1}{I} \oint\left(\boldsymbol{A}_{\mathrm{C} 1}+\boldsymbol{A}_{\mathrm{D} 1}\right) \cdot \mathrm{d} \boldsymbol{l} \\
& =L_{\mathrm{p} 11}^{\prime}+L_{\mathrm{p} 12}^{\prime}+L_{\mathrm{p} 13}^{\prime}+L_{\mathrm{p} 14}^{\prime} \\
& =\frac{1}{I} \sum_{i=1}^{4} \int_{\mathrm{S} i}\left(\boldsymbol{A}_{\mathrm{C} 1}+\boldsymbol{A}_{\mathrm{D} 1}\right) \cdot \mathrm{d} \boldsymbol{l}
\end{aligned}
$$

Where $\boldsymbol{A}_{\mathrm{C} 1}$ and $\boldsymbol{A}_{\mathrm{D} 1}$ are generated by segment S1. $L_{\mathrm{p} i j}^{\prime}$ represents mutual partial inductance between segment $\mathrm{S} i$ and $\mathrm{S} j$. When $i=j, L_{\mathrm{p} i j}^{\prime}$ means self partial inductance of segment $\mathrm{S} i$. Therefore, inductance of segment $\mathrm{S} 1$ can be calculated by

$$
L_{\mathrm{p} 11}^{\prime}=\frac{1}{I} \int_{\mathrm{S} 1} \boldsymbol{A}_{\mathrm{C} 1} \cdot \mathrm{d} \boldsymbol{l}+\frac{1}{I} \int_{\mathrm{S} 1} \boldsymbol{A}_{\mathrm{D} 1} \cdot \mathrm{d} \boldsymbol{l}
$$

As mentioned above, $\boldsymbol{A}_{\mathrm{D} 1}$ is irrotational, i.e., the second integral is merely related to locations of head and end points in path $\mathrm{S} 1$, leading to a convenient calculation.

Similarly, mutual partial inductance between two segments $\mathrm{S} i$ and $\mathrm{S} j$ is defined by

$$
L_{\mathrm{p} i j}^{\prime}=\frac{1}{I} \int_{\mathrm{S} j} \boldsymbol{A}_{\mathrm{C} i j} \cdot \mathrm{d} \boldsymbol{l}+\frac{1}{I} \int_{\mathrm{S} j} \boldsymbol{A}_{\mathrm{D} i j} \cdot \mathrm{d} \boldsymbol{l}
$$

where $\boldsymbol{A}_{\mathrm{C} i j}$ and $\boldsymbol{A}_{\mathrm{D} i j}$ are along path $\mathrm{S} j$ and are generated by segment $\mathrm{S} i$.

\section{Numerical Experiment for Comparison}

A simplified structure based on practical module, as shown in Fig. 4, is used for numerical experiment and comparison of proposed method and existing method.

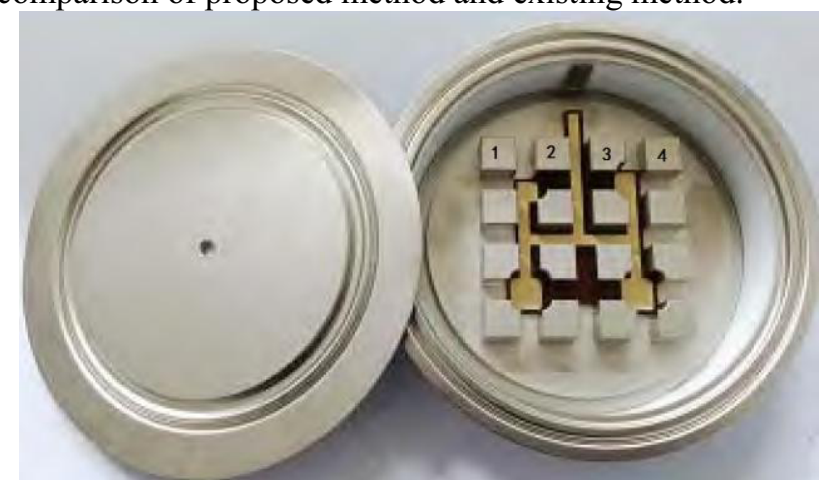

Fig. 4. Copper emitter pillars layout of press-pack IGBT

Inductance due to pillars is important part of stray inductance of package and has obvious influence on current distribution [9], hence we compare the partial inductance of pillars using both proposed method and existing method. The calculated model is shown in Fig.5.

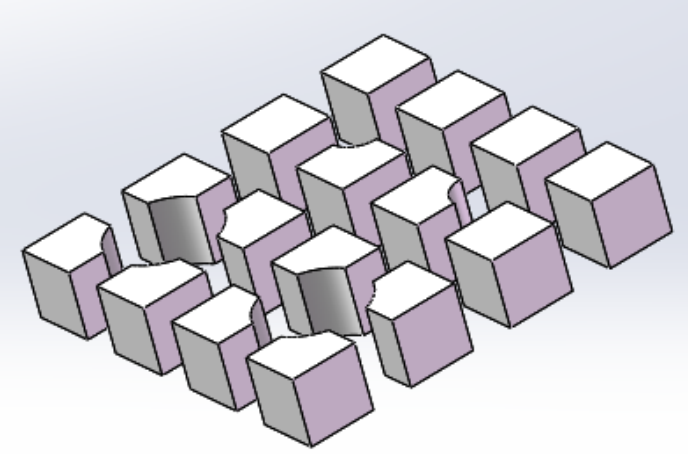

Fig. 5. Calculated model

We make one simplification that the notches of pedestals are neglected, so all of pedestals are cuboid. Self-inductance and mutual-inductance of pillars are calculated and shown followed.

Table. 1. Comparison of partial inductance calculation using existing method and proposed method

\begin{tabular}{lcc}
\hline Partial Inductance & Existing method & Proposed method \\
\hline Self-L of 1 & $1.83 \mathrm{nH}$ & $1.24 \mathrm{nH}$ \\
Mutual-L of 1\&2 & $0.665 \mathrm{nH}$ & $0.362 \mathrm{nH}$ \\
Mutual-L of 1\&3 & $0.337 \mathrm{nH}$ & $0.172 \mathrm{nH}$ \\
Mutual-L of 1\&4 & $0.225 \mathrm{nH}$ & $0.114 \mathrm{nH}$ \\
\hline
\end{tabular}

Such 16 pillars lead to 256 results of inductance, so typical results are picked and shown in Table. 1. Results 
show that displacement current obviously affect calculation of partial inductance in practical situation.

The influence caused by inductance extraction is analyzed by simulation of IGBT current calculation. The electrical structures of press-pack IGBT in Fig.4 is shown followed.

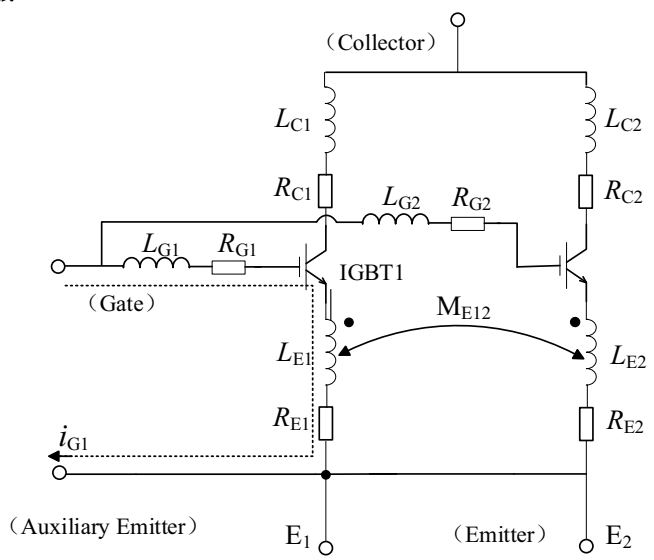

Fig. 5. Calculated model

where $L_{C 1}, L_{C 2}, R_{C 1}, R_{C 2}$ represent collector inductance and collector resistance for IGBT1 and IGBT2 separately, $L_{E 1}, L_{E 2}, R_{E 1}, R_{E 2}$ are emitter inductance and emitter resistance, $L_{G 1}, L_{G 2}, R_{G 1}, R_{G 2}$ represent inductance and collector resistance of gate side. $\mathrm{M}_{E 12}$ represents mutual inductance of emitter side.

Simulation and experiment are made for comparing above two methods. Result of current is shown followed.

Table. 2. Result of current

\begin{tabular}{cccc}
\hline $\begin{array}{c}\text { Current } \\
\text { peak }\end{array}$ & $\begin{array}{c}\text { Existing } \\
\text { method }\end{array}$ & $\begin{array}{c}\text { Proposed } \\
\text { method }\end{array}$ & $\begin{array}{c}\text { Experiment } \\
\text { Measurement }\end{array}$ \\
\hline Chip 1 & 7.49 & 7.58 & 8.10 \\
Chip 2 & 21.4 & 21.6 & 22.6 \\
\hline
\end{tabular}

Results show that proposed method could improve the calculation accuracy of simulation. The influence will be obvious whereas IGBT module become larger.

\section{Conclusion}

Precious inductance extraction of conductor is important in analysis of electrical performance. This paper proposes a method of inductance extraction for press-pack IGBT where conductor segments might not form a closed loop. By considering displacement current, the conductor segment model could meet ACL. Vector potential $\boldsymbol{A}$ also could be affected by displacement current, which leads to a different formulation of partial inductance. A simplified model based on practical structure are used in numerical experiment, and obtains different results of existing method and proposed method.

\section{Acknowledgement}

This work was supported by the 02 Support Project: Research on key technology of IGBT with high short circuit shutdown capability (SGRI-GB-71-16-003)

\section{References}

1. A. E. Ruehli, "Inductance Calculations in a Complex Integrated Circuit Environment," IBM Journal of Research and Development, vol. 16, no. 5, pp. 470-481, Sept. 1972.

2. Paul C R, "The Concept of Partial Inductance," in Inductance: loop and partial, New Jersey: John Wiley \& Sons, 2011, pp. 195-245.

3. Holloway C L, Kuester E F, Ruehli A E, et al. "Partial and internal inductance: Two of Clayton R. Paul's many passions," Electromagnetic Compatibility, IEEE Transactions on, vol. 55, no. 4, pp. 600-613, 2013.

4. Cao Y S, Jiang L J, Ruehli A E. "Distributive radiation and transfer characterization based on the PEEC method," Electromagnetic Compatibility, IEEE Transactions on, vol. 57, no. 4, pp. 734-742, 2015.

5. Antonini G, Romano D. "Adaptive-cross-approximation-based acceleration of transient analysis of quasi-static partial element equivalent circuits," Microwaves, Antennas \& Propagation, IET, vol. 9, no. 7, pp. 700-709, 2015.

6. Hackl Y, Scholz P, Ackermann W, et al. "Multifunction Approach and Specialized Numerical Integration Algorithms for Fast Inductance Evaluations in Nonorthogonal PEEC Systems," Electromagnetic Compatibility, IEEE Transactions on, vol. 57, no. 5, pp. 1155-1163, 2015.

7. Kalhor H. "Comparison of Ampere's circuital law (ACL) and the law of Biot-Savart (LBS)," Education, IEEE Transactions on, vol. 31, no. 3, pp. 236-238, 1988.

8. Kalhor H. "The degree of intelligence of the law of Biot-Savart," Education, IEEE Transactions on, vol. 33, no. 4, pp. 365-366, 1990.

9. TANG Xinling, CUI Xiang, ZHAO Zhibin, et al, "Analysis of Transient Current Distribution Characteristics of Parallel Chips in Press Pack IGBT," Proceedings of the CSEE, vol. 36, pp. 1-11, 2016 (in Chinese). 